\title{
ARTICLE OPEN Atomistic material behavior at extreme pressures
}

\author{
Laurent Karim Béland ${ }^{1}$, Yuri N Osetsky ${ }^{1}$ and Roger E Stoller ${ }^{1}$
}

Computer simulations are routinely performed to model the response of materials to extreme environments, such as neutron (or ion) irradiation. The latter involves high-energy collisions from which a recoiling atom creates a so-called atomic displacement cascade. These cascades involve coordinated motion of atoms in the form of supersonic shockwaves. These shockwaves are characterized by local atomic pressures $>15 \mathrm{GPa}$ and interatomic distances $<2 \AA$. Similar pressures and interatomic distances are observed in other extreme environment, including short-pulse laser ablation, high-impact ballistic collisions and diamond anvil cells. Displacement cascade simulations using four different force fields, with initial kinetic energies ranging from 1 to $40 \mathrm{keV}$, show that there is a direct relationship between these high-pressure states and stable defect production. An important shortcoming in the modeling of interatomic interactions at these short distances, which in turn determines final defect production, is brought to light.

npj Computational Materials (2016) 2, 16007; doi:10.1038/npjcompumats.2016.7; published online 5 August 2016

\section{INTRODUCTION}

As scientific computing becomes evermore ubiquitous, it is common practice to simulate the effect of extreme environments on materials using molecular dynamics (MD). The ability to capture a material's response atom by atom has helped understand and complement experiments. For example, thanks to $M D$, the thermodynamic processes that control the production of nanoparticules after pulsed laser irradiation are relatively well understood. ${ }^{1}$ Notably, such computational results explain the Newton rings observed in experiments. ${ }^{2,3} \mathrm{MD}$ also led to a better understanding of the nature of the destructive shockwaves that follow high-energy impact ${ }^{4-6}$ and the behavior of materials at high pressure in diamond anvil cells. ${ }^{7-9}$ It can predict many properties, such as the Hugoniot, dislocation densities after impact and fracture behavior. In the case of neutron and ion irradiation, MD can be used to predict the evolution of ballistic collisions that occur, and the nature of primary damage produced by the high-energy recoils. ${ }^{10}$ It can also shed light on a plethora of mechanisms that take place as the kinetic energy of the recoil is dissipated: fractal replacement sequences, ${ }^{11}$ supersonic shockwaves, ${ }^{12}$ sonic waves, ${ }^{12}$ the formation of liquid-like regions and their recrystallization or amorphization. ${ }^{13}$

The aim of many MD studies is to describe the mechanisms and general trends, not to make precise predictions. However, as the scientific community moves towards materials by design and systematic computational characterization, increasingly precise and accurate models are required. In the case of atomistic modeling, methods that explicitly account for electronic structure, such as $a b$ initio MD, are considered the gold standard. Unfortunately, their use has very serious limitations, given their high computational cost. In particular, the simulation of high-energy perturbations such as those involved in neutron or ion irradiation requires large simulation cells: a few hundred thousands of atoms at a minimum, but sometimes reaching billions of atoms. Most electronic structure codes do not scale well past a few hundred atoms, especially if modeling metals.
In addition, the high initial atomic velocities involved in these simulations limit timesteps to a fraction of those used in equilibrium MD $\left(\sim 10^{-18} \mathrm{~s}\right.$ rather than $\left.\sim 10^{-15} \mathrm{~s}\right)$. For these reasons, MD based on classical (or semi-empirical) force fields is currently the only technique used to simulate these energetic events.

These classical force fields are generally parameterized to obtain agreement with equilibrium and near-equilibrium properties, such as elastic constants, phonon spectra, phase transitions, and point-defect formation and migration energies. Given the high velocities involved, atoms move much closer to each other than under equilibrium conditions. Figure 1a illustrates this point for iron. The shortest atom-to-atom distance in a displacement cascade is plotted as a function of the energy of the primary knock-on atom (PKA). Distances approaching $0.7 \AA$ A, i.e., 0.24 of the equilibrium lattice parameter, are observed. To adequately model the interaction energies at these short distances, one cannot extrapolate the force field based on equilibrium properties. For very-short-distance interactions (less than $\sim 1.2 \AA)$, it is common practice to use the 'universal' screened Coulomb force fields developed by Ziegler, Biersack and Littmark, which were calibrated using Hartree-Fock calculations of two atoms in vacuum. ${ }^{14}$ The potential energy and forces for distances between $1.2 \AA$ and near-equilibrium distances of $\sim 2 \AA$ are obtained from an arbitrary interpolation of the equilibrium and Ziegler, Biersack and Littmark force fields. The potential energies corresponding to these short distances are given for the case of Fe in the secondary $y$ axis of Figure $1 \mathrm{a}$, where values as high as $210 \mathrm{eV}$ per atom are seen for the case of a 0.9-Å separation.

The transition from the supersonic shockwave regime to the sonic regime is of particular interest. Indeed, the supersonic shockwave is considered destructive, whereas the sonic is considered non-destructive. ${ }^{12}$ The former involves high kinetic energies at the shockwave front, larger than the displacement energy threshold. In other words, these atoms have enough momentum to potentially permanently dislodge their neighbors

\footnotetext{
${ }^{1}$ Materials Science and Technology Division, Oak Ridge National Laboratory, Oak Ridge, TN, USA.

Correspondence: LK Béland (belandlk@ornl.gov)

Received 19 January 2016; revised 7 March 2016; accepted 11 April 2016 

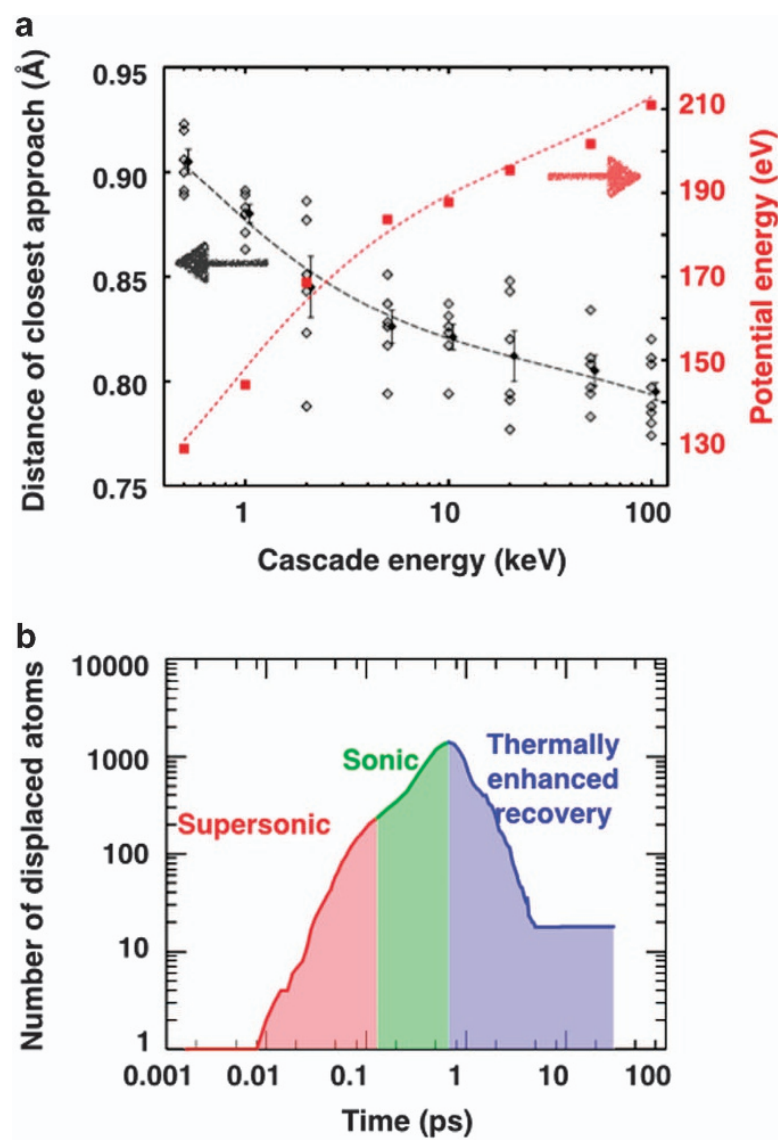

Figure 1. (a) Distance of closest approach and corresponding potential energy for iron atoms as a function of atomic recoil energy. Black dots are the average values obtained from the individual simulations shown as open diamonds. The s.e.m. is indicated by the error bars, with the scatter arising from random atomic velocities in the target. The red squares are the potential energy associated with the average distance. The dashed lines are guides for the eye. (b) The time evolution of the number of displaced atoms, identified using Wigner-Seitz cell analysis, during a 10-keV displacement cascade in $\mathrm{Ni}$ at $300 \mathrm{~K}$; three phases are shown: supersonic, sonic and thermally enhanced recovery.

from their original lattice sites. The later involves primarily small elastic displacements, which eventually lead the atoms back to their original lattice position. Characterizing the system at the point in time of this transition should provide insights into the nature of primary damage formation.

In this report, the results of simulations involving energetic knock-ons in $\mathrm{Ni}$ are presented and analyzed. The appearance of coordinated atom motion, leading to a supersonic shockwave, is presented and characterized. It is shown that the displacements created by this shockwave are highly correlated with the number of final stable defects created by the PKA in the Ni crystal. At this stage of cascade development, the local material state is described by the least accurate part of classical force fields. Furthermore, the crucial importance of correctly modeling short-range interatomic interactions is highlighted.

\section{RESULTS}

The evolution of high-energy displacement cascades can conveniently be subdivided into three distinct phases: a supersonic phase, a sonic phase and a thermally enhanced recovery phase. ${ }^{12} \mathrm{~A}$ typical time profile of the number of atomic displacements is plotted in Figure $1 \mathrm{~b}$. The three phases are indicated by different colors. Note that the transition from the supersonic regime to the sonic regime is associated with an inflection point in Figure 1b. At this moment in time, the fastest atom in the system typically has a kinetic energy lower than that of the 'displacement threshold' of $\sim 40 \mathrm{eV}$. As a result, beyond this time, atoms no longer have sufficient energy to permanently displace a neighbor from its lattice site. Therefore, the increasing number of atoms displaced during the sonic regime are short-lived transient defects, which will recover their original lattice position shortly thereafter. This observation is consistent with those of Calder et al. ${ }^{12}$

A noticeable feature of the supersonic regime is that the PKA creates many secondary and higher generation knock-ons. The coordinated motion of these energetic atoms creates a supersonic pressure wave. This is illustrated in Figure 2. The pressure waves are easily identifiable using the local atomic stress or atomic volume. Three moments in time are illustrated: at 0.10 , 0.15 and $0.25 \mathrm{ps}$ after cascade initiation. These correspond to times before, during and after the supersonic to sonic transition. The pressure wavefront is characterized by small atomic volumes and high local pressure (i.e., black spots in Figure 2). The supersonic wave leaves behind a region of high atomic volume and small or negative local pressure. In this cross-section, one can identify two interacting shockwaves. The low-density pockets are well known to be precursors of vacancy-type defects formed at the end of cascade evolution, and the interaction of nearby shockwaves is known to produce large interstitial-type clusters. ${ }^{12}$ Likewise, the interactions of these shockwaves with a free surface can lead to the surface roughening and creation of huge vacancy dislocation loops. ${ }^{15,16}$ In the 0.25 -ps snapshot, one can see that the sonic wave propagates without creating low-density regions. For this reason, the supersonic regime is often considered destructive, whereas the sonic regime is considered non-destructive. Finally, during the thermally enhanced recovery regime, the highly disordered region created by the supersonic wave cools down and recrystallizes. The atoms displaced by the sonic wave recover their original positions. During this phase, temperatures can be very high in the core of the cascade (reaching over $4,500 \mathrm{~K}$ in the low-density pockets of our $10 \mathrm{keV}$ simulations); atoms that were displaced during the supersonic phase remain very mobile and many recombine with vacant lattice sites. By $\sim 10-20 \mathrm{ps}$, thermal equilibrium is restored and a number of stable atomic displacements (vacant lattice sites and atoms in interstitial positions) remain. A complete sequence of snapshots illustrated in Figure 2 can be found in Supplementary Figure S1 in the Supplementary Materials, along with Supplementary Movie S2-S4.

In Figure 3, the correlation between the number of atoms displaced at the end of the supersonic stage and the number of stable defects at the end of the cascade is demonstrated. The dashed line is the best linear fit to the data. Each data point is averaged over 16 runs, using a given initial PKA energy $(1,5,10,20$ or $40 \mathrm{keV})$ and force field (data obtained using two $\mathrm{Ni}$ force fields and a $\mathrm{Ni}-\mathrm{Co}$ and $\mathrm{Ni}-\mathrm{Fe}$ force field). The trend is very clear. It is possible to predict, on average, how many stable defects will be present in the system by measuring the number of atoms displaced by the supersonic wave. This shows the crucial importance of correctly modeling these supersonic waves. One should note that this is not only a linear log-log relationship, but a true proportionality. In other words, the line in Figure 3 is a power law with an exponent of 1.

The magnitude of the stress involved in these waves is remarkable. As shown in Figure 2, some atoms exhibit local pressures as high as $50 \mathrm{GPa}$ in $10 \mathrm{keV}$ cascades. Such pressures are comparable to those observed during short-pulse laser ablation, high-energy impacts and diamond anvil cells. The interatomic distances involved are equally remarkable features of this process. As shown in the Supplementary Materials, they are typically 

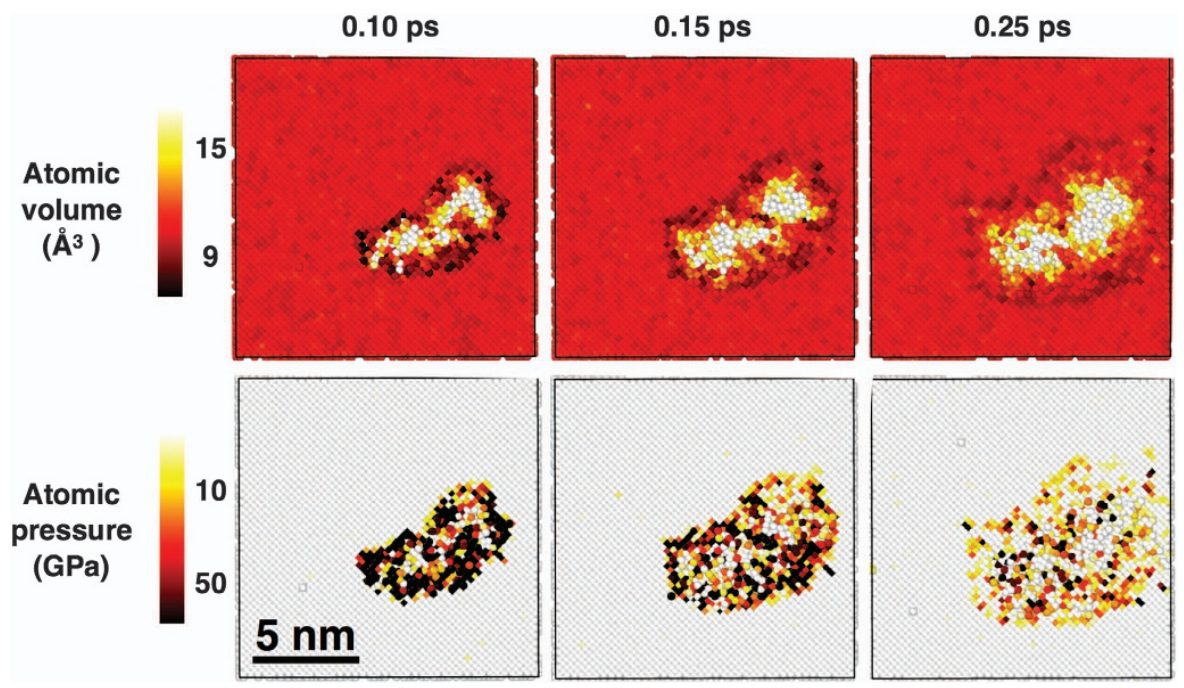

Figure 2. Snapshots of atomic volume and local atomic pressure during a $10-\mathrm{keV}$ displacement cascades in $\mathrm{Ni}$, at times $0.1,0.15$ and $0.25 \mathrm{ps}$ after the creation of a PKA. A 5-atom-thick cross-section of a 256,000-atom system is shown. These times correspond to the transition between the supersonic and sonic phase.

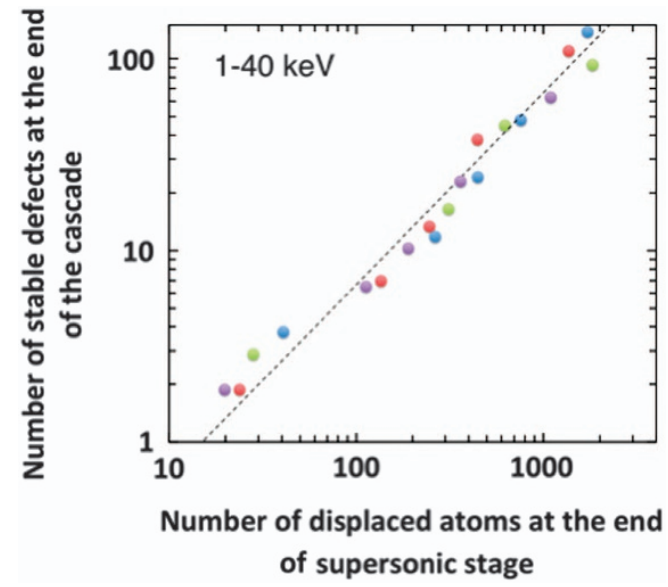

Figure 3. Near-linear relationship between the number of atoms displaced at the end of the supersonic stage and the final number of stable defects $\left(R^{2}=0.93\right)$. It is important to note that the power law in this log-log plot has an exponent of 1. Data obtained from cascades with $1,5,10,20$ and $40 \mathrm{keV}$ of initial kinetic energy; different colors indicate different classical potentials.

between 1.4 and $2.0 \AA$ (0.4-0.6 of the lattice parameter) in the high-density, high-pressure zone surrounding the low-density pockets. In other words, the atomic displacements created by the supersonic wave are determined by the energetics of interatomic interactions at these short distances.

\section{DISCUSSION}

The simulations herein exhibit extreme conditions. They raise serious questions about the accuracy of results obtained with the force fields used by the scientific community to model such highenergy phenomena. Indeed, as explained in the introduction, the parameterization of these potentials in the critical between 1.2 and $2.0 \AA$ is very loosely constrained in practice. However, these are precisely the distances involved in these high-pressure processes. In the case of displacement cascades, Figure 3 shows that configurations involving short distances are directly related with final defect production. Although current modeling techniques are probably adequate to identify general features of these high-energy phenomena, our analysis indicates that the force fields used to compute short-range interactions require more physically based constraints to generate quantitatively accurate simulation results. It is also important to note that the two Ni potentials showcased in this study ${ }^{17,18}$ have very similar equilibrium properties, but nonetheless exhibit different number of stable defects. Likewise, both $\mathrm{Ni}$ potentials and their respective alloys (Mishin's $\mathrm{Ni}-\mathrm{NiCo}^{18,19}$ and Bonny's $\mathrm{Ni}-\mathrm{NiFe}^{17}$ ) have largely similar interstitial formation energies and displacement energy thresholds. Using the Mishin potentials, the $\langle 100\rangle$ dumbbell interstitial formation energy is $3.97 \mathrm{eV}$ in $\mathrm{Ni}$ and has a range of $3.8-4.2 \mathrm{eV}$ in NiCo. For the Bonny potentials, the $\langle 100\rangle$ dumbbell interstitial formation energy is $5.83 \mathrm{eV}$ in $\mathrm{Ni}$ and has a range of $4.5-7.5 \mathrm{eV}$ in NiFe. For displacement thresholds, the respective values are 37, 40, 55 and $51 \mathrm{eV}$. The divergence in these values is not enough to explain the divergence in the number of stable defects produced by each potential. This suggests that the variations in stable defect production from one potential to another are largely due to short-range interactions between 1.2 and $2.0 \AA$.

Formally, the effect of these short-term interactions on the final outcome was only shown for the case of neutron and ion irradiation. However, the pressures involved are comparable to those of other high-energy processes, such as laser ablation, high-energy impact and diamond anvil cells. ${ }^{1-9}$ This suggests that further work is needed to improve the description of atomic force fields at short interatomic distances in order for future computational work using these force fields to be as quantitatively accurate as possible. Some efforts have previously been made in this direction. For example, Tiwary et $a l^{20}$ took special precautions to ensure that their uranium oxide potential was based on the $a b$ initio data at these distances. Zhakhovskii et al. ${ }^{21}$ showed that high-tension states should also be included in the fitting database of an embedded atom method (EAM) potential to properly describe short-pulse laser ablation. Likewise, Zhang el al. ${ }^{22}$ demonstrated that properly reproducing generalized stacking fault energies is of crucial importance to model compressive shocks. Our study strongly indicates that such efforts are justified and should be pursued in a systematic fashion.

\section{MATERIALS AND METHODS}

Displacement cascades with $10-40 \mathrm{keV}$ of initial kinetic energy were simulated; this energy range is relevant to the knock-ons produced during neutron or MeV ion-beam irradiation. MD simulations were performed 
using the LAMMPS package ${ }^{23}$ in an atomic system thermally equilibrated at $300 \mathrm{~K}$. The PKA was given initial velocity in the [135] direction. A variable timestep was used to maintain accurate integration of the equations of atomic motion. Interatomic interactions were modeled using four force fields: two for $\mathrm{Ni}^{17,18} \mathrm{NiFe}^{17}$ and $\mathrm{NiCo}{ }^{18,19}$ The number of displaced atoms was determined using a Wigner-Seitz cell analysis. Local pressure was computed using the trace of the atomic virial stress tensor and dividing by the atoms's Voronoi volume. The Voronoi volume calculation and vizualisation are performed with the Ovito package. ${ }^{24}$

\section{ACKNOWLEDGEMENTS}

We thank Valentino Cooper and William J Weber for reviewing the manuscript. This work was supported as part of the Energy Dissipation to Defect Evolution (EDDE), an Energy Frontier Research Center funded by the US Department of Energy, Office of Science, Basic Energy Sciences. LKB acknowledges additional support from a fellowship awarded by the Fonds Québécois de recherche Nature et Technologies. This manuscript has been authored by UT-Battelle, LLC under contract no. DE-AC05-00OR22725 with the US Department of Energy. The US Government retains and the publisher, by accepting the article for publication, acknowledges that the US Government retains a non-exclusive, paid-up, irrevocable, world-wide license to publish or reproduce the published form of this manuscript, or allow others to do so, for US Government purposes. The Department of Energy will provide public access to these results of federally sponsored research in accordance with the DOE Public Access Plan (http://energy.gov/downloads/doe-public-access-plan).

\section{CONTRIBUTIONS}

L.K.B. performed the majority of the simulations and drafted the manuscript. Y.N.O. and R.E.S. supervised the work and significantly edited the manuscript.

\section{COMPETING INTERESTS}

The authors declare no conflict of interest.

\section{REFERENCES}

1. Perez D. \& Lewis L. J. Ablation of solids under femtosecond laser pulses. Phys. Rev. Lett. 89, 255504 (2002).

2. Perez, D. \& Lewis, L. J. Molecular-dynamics study of ablation of solids under femtosecond laser pulses. Phys. Rev. B 67, 184102 (2003).

3. Sokolowski-Tinten, K. et al. Transient states of matter during short pulse laser ablation. Phys. Rev. Lett. 81, 224 (1998).

4. Kadau, K., Germann, T. C., Lomdahl, P. S. \& Holian, B. L. Microscopic view of structural phase transitions induced by shock waves. Science 296, 1681-1684 (2002).

5. Kadau, K. et al. Shock waves in polycrystalline iron. Phys. Rev. Lett. 98, 135701 (2007).

6. Holian, B. L. \& Lomdahl, P. S. Plasticity induced by shock waves in nonequilibrium molecular-dynamics simulations. Science 280, 2085-2088 (1998).

7. Zeng, Q. et al. Long-range topological order in metallic glass. Science $\mathbf{3 3 2}$, 1404-1406 (2011).

8. Murakami, M., Hirose, K., Kawamura, K., Sata, N. \& Ohishi, Y. Post-perovskite phase transition in MgSiO3. Science 304, 855-858 (2004).

9. Errandonea, D. Transition metals: can metals be a liquid glass? Nat. Mater. 8 , 170-171 (2009).

10. Stoller R. E. Comprehensive Nuclear Materials 293-332 (Elsevier, Amsterdam, Netherlands, 2012).

11. Kun, F. \& Bardos, G. Fractal dimension of collision cascades. Phys. Rev. E 55, 1508 (1997).

12. Calder, A. F., Bacon, D. J., Barashev, A. V. \& Osetsky, Y. N. On the origin of large interstitial clusters in displacement cascades. Philos. Mag. 90, 863-884 (2010).

13. Meldrum, S. J. Z., Boatner, L. A. \& Ewing, R. C. A transient liquid-like phase in the displacement cascades of zircon, hafnon and thorite. Nature 395 , 56-58 (1998).

14. Ziegler J. F., Biersack J. P. \& Littmark U. The Stopping and Range of lons in Matter Vol 1 (Springer US, Pergamon, 1985).

15. Nordlund, K., Keinonen, J., Ghaly, M. \& Averback, R. S. Coherent displacement of atoms during ion irradiation. Nature 398, 49-51 (1999).

16. Osetsky, Y. N., Calder, A. F. \& Stoller, R. E. How do energetic ions damage metallic surfaces? Curr. Opin. Solid State Mater. Sci. 19, 277-286 (2015).

17. Bonny, G., Castin, N. \& Terentyev, D. Interatomic potential for studying ageing under irradiation in stainless steels: the FeNiCr model alloy. Modell. Simul. Mater. Sci. Eng. 21, 085004 (2013).

18. Mishin, Y. Atomistic modeling of the $\gamma$ and $\gamma$-phases of the Ni-Al system. Acta Mater. 52, 1451-1467 (2004).

19. Purja Pun, G. P. \& Mishin, Y. Embedded-atom potential for hcp and fcc cobalt. Phys. Rev. B 86, 134116 (2012).

20. Tiwary, P., van de Walle, A. \& Gronbech-Jensen, N. Ab initio construction of interatomic potentials for uranium dioxide across all interatomic distances. Phys. Rev. B 80, 174302 (2009).

21. Zhakhovskii, V. V., Inogamov, N. A., Petrov, Y. V., Ashitkov, S. I. \& Nishihara, K. Molecular dynamics simulation of femtosecond ablation and spallation with different interatomic potentials. Appl. Surf. Sci. 225, 9592-9596 (2009).

22. Zhang, R. F., Wang, J., Beyerlein, I. J. \& Germann, T. C. Twinning in bcc metals under shock loading: a challenge to empirical potentials. Philos. Mag. Lett. 91, 731-740 (2011).

23. Plimpton, S. Fast parallel algorithms for short-range molecular dynamics. J. Comput. Phys. 117, 1-19 (1995).

24. Stukowski, A. Visualization and analysis of atomistic simulation data with OVITOthe Open Visualization Tool. Modell. Simul. Mater. Sci. Eng. 18, 015012 (2010).

\section{(c) (i)}

This work is licensed under a Creative Commons Attribution 4.0 International License. The images or other third party material in this article are included in the article's Creative Commons license, unless indicated otherwise in the credit line; if the material is not included under the Creative Commons license, users will need to obtain permission from the license holder to reproduce the material. To view a copy of this license, visit http://creativecommons.org/licenses/ by/4.0/

Supplementary Information accompanies the paper on the npj Computational Materials website (http://www.nature.com/npjcompumats) 